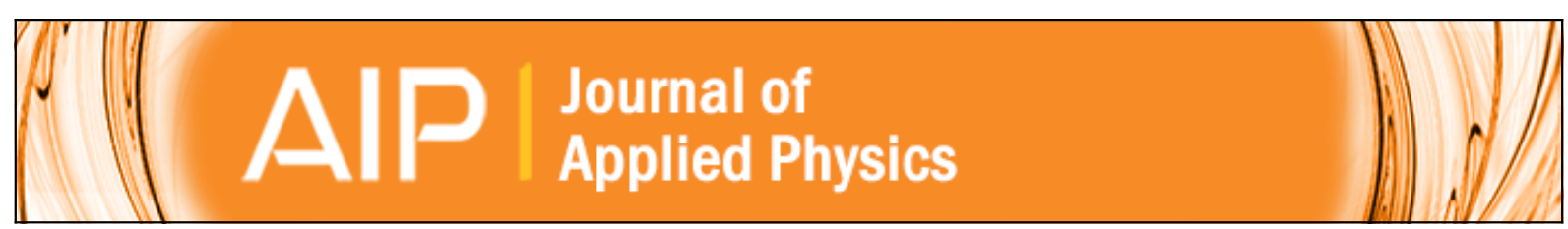

Thin SiGe virtual substrates for Ge heterostructures integration on silicon

S. Cecchi, E. Gatti, D. Chrastina, J. Frigerio, E. Müller Gubler, D. J. Paul, M. Guzzi, and G. Isella

Citation: Journal of Applied Physics 115, 093502 (2014); doi: 10.1063/1.4867368

View online: http://dx.doi.org/10.1063/1.4867368

View Table of Contents: http://scitation.aip.org/content/aip/journal/jap/115/9?ver=pdfcov

Published by the AIP Publishing

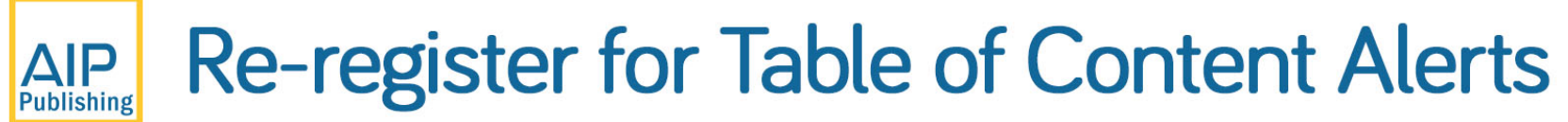




\title{
Thin SiGe virtual substrates for Ge heterostructures integration on silicon
}

\author{
S. Cecchi, ${ }^{1}{ }^{1, a)}$ E. Gatti, ${ }^{2}$ D. Chrastina, ${ }^{1}$ J. Frigerio, ${ }^{1}$ E. Müller Gubler, ${ }^{3}$ D. J. Paul, ${ }^{4}$ M. Guzzi, ${ }^{2}$ \\ and G. Isella ${ }^{1}$ \\ ${ }^{1}$ L-NESS, Dipartimento di Fisica, Politecnico di Milano-Polo Territoriale di Como, Via Anzani 42, \\ I-22100 Como, Italy \\ ${ }^{2}$ L-NESS, Dipartimento di Scienza dei Materiali, Università di Milano Bicocca, via Cozzi 53, I-20126 Milano, \\ Italy \\ ${ }^{3}$ Electron Microscopy ETH Zurich, ETH Zurich, Auguste-Piccard-Hof 1, CH-8093 Zurich, Switzerland \\ ${ }^{4}$ School of Engineering, University of Glasgow, Rankine Building, Oakfield Avenue, Glasgow G12 8LT, \\ United Kingdom
}

(Received 28 November 2013; accepted 20 February 2014; published online 4 March 2014)

\begin{abstract}
The possibility to reduce the thickness of the SiGe virtual substrate, required for the integration of Ge heterostructures on $\mathrm{Si}$, without heavily affecting the crystal quality is becoming fundamental in several applications. In this work, we present $1 \mu$ m thick $\mathrm{Si}_{1-x} \mathrm{Ge}_{x}$ buffers (with $x>0.7$ ) having different designs which could be suitable for applications requiring a thin virtual substrate. The rationale is to reduce the lattice mismatch at the interface with the Si substrate by introducing composition steps and/or partial grading. The relatively low growth temperature $\left(475^{\circ} \mathrm{C}\right)$ makes this approach appealing for complementary metal-oxide-semiconductor integration. For all the investigated designs, a reduction of the threading dislocation density compared to constant composition $\mathrm{Si}_{1-x} \mathrm{Ge}_{x}$ layers was observed. The best buffer in terms of defects reduction was used as a virtual substrate for the deposition of a $\mathrm{Ge} / \mathrm{SiGe}$ multiple quantum well structure. Room temperature optical absorption and photoluminescence analysis performed on nominally identical quantum wells grown on both a thick graded virtual substrate and the selected thin buffer demonstrates a comparable optical quality, confirming the effectiveness of the proposed approach. (C) 2014 AIP Publishing LLC. [http://dx.doi.org/10.1063/1.4867368]
\end{abstract}

\section{INTRODUCTION}

The development of Ge-compatible high- $\kappa$ dielectrics has recently renewed the interest in the epitaxial growth of high Ge content heterostructures for advanced MOSFET fabrication. ${ }^{1}$ Moreover, Ge is gaining a great deal of attention in relevant scientific and technological areas, spanning from photonics to spintronics and energy harvesting. Electroabsorption modulation (Refs. 2-4), as well as light detection and emission (Ref. 5) based on Ge quantum wells (QWs), have been demonstrated, and optical spin orientation and detection has been recently achieved in similar heterostructures. ${ }^{6}$ The application of Ge QWs for thermoelectric energy generation is also under investigation. ${ }^{7,8}$ All these applications rely on the deposition of high quality epitaxial material and engineered low dimensional structures on $\mathrm{Si}$.

Since the lattice mismatch between $\mathrm{SiGe}$ and $\mathrm{Si}$ reaches $4 \%$ for pure $\mathrm{Ge}$, the integration of Ge-rich SiGe heterostructures on Si requires the insertion of a buffer layer meant to plastically accommodate the mismatch strain. The relaxation process occurs with the introduction of misfit dislocations (MD) at the interface. Threading arms extending from the $\mathrm{SiGe} / \mathrm{Si}$ interface to the surface act as scattering and recombination centers for carriers and are thus detrimental for the performances of electronic and optoelectronics devices. ${ }^{9,10}$

\footnotetext{
${ }^{a)}$ Electronic mail: stefano.cecchi@mdm.imm.cnr.it. Present address: Laboratorio MDM, CNR-IMM-Unità di Agrate Brianza, Via Olivetti 2, I-20041 Agrate Brianza, Italy.
}

The thermoelectric properties can also be negatively affected by an excessive density of threading dislocations (TD). ${ }^{11}$

For a given lattice mismatch $f=\left(a_{0}-a_{f}\right) / a_{f}$ (where $a_{f}$ and $a_{0}$ are the bulk lattice constants of the film and the substrate, respectively), the misfit dislocation density required to relax a SiGe epilayer is given by the relation $|f|=b^{\prime} / p$, where $b^{\prime}$ is the effective Burgers vector and $1 / p$ is the number of MD per unit length. ${ }^{12}$ The threading dislocation density (TDD) is, instead, related to the length of the misfit segments at the heterointerface with no simple relation to the degree of relaxation. In $\mathrm{SiGe} / \mathrm{Si}$ heteroepitaxy, the TDD is experimentally seen to increase with lattice mismatch (see Ref. 13 for a collection of data on the TDD dependence on $f$ ). For constant composition $\mathrm{Si}_{1-x} \mathrm{Ge}_{x}$ layers with $x>0.6$ directly grown on Si the TDD can reach $10^{9} \mathrm{~cm}^{-2} .{ }^{13}$

A well established approach allowing the reduction of the TDD consists in linearly grading the Ge content in the buffer layer from pure Si to the final alloy composition $x_{f}{ }^{14}$ The continuous reduction of substrate temperature during the growth minimizes surface roughening, making intermediate chemical mechanical polishing (CMP) steps unnecessary. ${ }^{15}$ The composition grading, by leading to a partialization of the misfit strain along the layer, allows MD to be nucleated gradually and enhances dislocation glide, promoting the formation of longer misfit segments and, hence, reducing the threading arm density. These graded layers are usually relatively thick with an optimal grading rate around $7 \%-10 \% / \mu \mathrm{m} .{ }^{16,17}$ For $x>0.6$, the resulting thickness is prohibitive for molecular beam epitaxy due to the relatively low 
deposition rates and solid source consumption. The attainment of TDD below $5 \times 10^{6} \mathrm{~cm}^{-2}$ requires an intermediate CMP step for virtual substrates (VSs) grown by ultra-high vacuum chemical vapor deposition (UHV-CVD) and reduced pressure-chemical vapor deposition (RP-CVD). ${ }^{18,19}$ A TDD $\sim 5 \times 10^{6} \mathrm{~cm}^{-2}$ is attainable for Ge-rich graded virtual substrates (GVS) grown by low-energy plasma-enhanced chemical vapor deposition (LEPECVD). ${ }^{20}$

A so called "reverse grading approach," where a reverse graded layer is grown on a relaxed Ge epilayer, has also been proposed..$^{21}$ Extremely high hole mobility has been demonstrated in modulation-doped $\mathrm{Ge}$ channels deposited on such a buffer. ${ }^{22}$ Nevertheless, the total thickness of these structures is above $2 \mu \mathrm{m}$, and the presence of the buried Ge layer can promote wafer bowing and possibly cracking (see Ref. 23) due to the mismatch of Ge and Si thermal expansion coefficients.

The possibility to reduce the thickness of the VS maintaining a good crystal quality becomes fundamental for many purposes, such as the integration of optical functionalities on complementary metal-oxide-semiconductor (CMOS) technology, the inhibition of parallel heat paths in lateral thermoelectric devices, and the enhancement of heat dispersion in vertical optoelectronic devices. Moreover, the use of thin relaxed buffers inhibits thermal strain related phenomena such as wafer cracking, bending, and exfoliation. ${ }^{24,25}$

In this work, we present an approach which could be suitable for applications requiring a reduced thickness VS. The strategy we followed is based on the attempt to adapt the thick GVS approach to a thin buffer layer. The rationale is to reduce the lattice mismatch at the interface with the $\mathrm{Si}$ substrate (and hence the threading dislocation formation at the initial stage of the relaxation) by introducing composition steps and/or partial grading (see Sec. II). Different designs are presented and compared, keeping constant the final composition and the total thickness of the buffer layer. In all cases, the chosen growth temperature guarantees a low thermal budget.

The different samples were structurally characterized by means of atomic force microscopy (AFM), high resolution X-ray diffraction (HRXRD) and transmission electron microscopy (TEM), and compared in terms of TDD (see Sec. III).

The buffer showing the lowest TDD was used as VS for the deposition of $\mathrm{Ge} / \mathrm{SiGe}$ multiple quantum well (MQW) structures; their optical quality was compared with that of analogous MQWs grown on thick GVSs, which can be considered a benchmark of high structural and optical quality (see Sec. IV).

\section{SAMPLES DESIGN AND EXPERIMENTAL}

In this study, $1 \mu \mathrm{m}$ thick buffers having a final Ge content $x_{f}=75 \%$ with different designs were considered. An additional $1 \mu$ m thick $\mathrm{Si}_{0.25} \mathrm{Ge}_{0.75}$ layer was deposited on top of all the buffers to better resolve the final Ge content by HRXRD. The samples were grown by LEPECVD on $\mathrm{Si}$ (001) substrates. The growth rate was optimized for each protocol as reported in Table I. The substrate temperature was fixed at $475^{\circ} \mathrm{C}$ in all the growth processes, which is the optimized growth temperature for MQWs in our system. No annealing step was performed. Figure 1 and Table I summarize the main structural properties of the different buffers.

Sample A (reference buffer) consists in a constant composition $\mathrm{Si}_{0.25} \mathrm{Ge}_{0.75}$ layer deposited at $6 \mathrm{~nm} / \mathrm{s}$. In samples $\mathrm{B}$, $\mathrm{C}$, and $\mathrm{D}$, the final composition $x_{f}$ of the relaxed $\mathrm{Si}_{1-x} \mathrm{Ge}_{x}$ buffer is reached by means of an intermediate $\mathrm{Si}_{1-y} \mathrm{Ge}_{y}$ layer with $y<x$.

Sample B (double step buffer) features an intermediate $0.5 \mu \mathrm{m}$ thick constant composition layer with a Ge content $y=40 \%$, which is the highest mismatch still guaranteeing a 2D growth. ${ }^{26}$ Since the substrate temperature was kept constant during all the deposition, this first step layer was grown using a reduced growth rate of $2 \mathrm{~nm} / \mathrm{s}$ to ensure a relatively good crystal quality. On top of this layer, a second layer having the final Ge content $x_{f}$ was deposited at a growth rate of $6 \mathrm{~nm} / \mathrm{s}$, as for sample A.

Sample C (double step graded buffer) is characterized by an intermediate $0.5 \mu \mathrm{m}$ thick constant composition layer with a Ge content $y=40 \%$ (growth rate of $2 \mathrm{~nm} / \mathrm{s}$ ), followed by a second layer linearly graded from $y$ to the final Ge content $x_{f}$ (grading rate $70 \% / \mu \mathrm{m}$ ). The growth rate of this graded layer was varied from $2.0 \mathrm{~nm} / \mathrm{s}$ (the same used for the $y=40 \%$ layer) to $1.2 \mathrm{~nm} / \mathrm{s}$, accordingly with the change in composition.

Sample D (partial graded buffer) presents a single linearly graded layer. Due to the difficulties in growing low $\mathrm{Ge}$ content alloys at low temperatures, the starting alloy composition $y^{\prime}$ was chosen to be $30 \%$. A grading rate of $45 \% / \mu \mathrm{m}$ is consequently obtained. In this case, the growth rate was varied from $10 \mathrm{~nm} / \mathrm{s}$ to $6 \mathrm{~nm} / \mathrm{s}$.

HRXRD measurements were performed using a PANalytical X'Pert PRO MRD high resolution X-ray diffractometer equipped with a hybrid mirror and a 2-bounce asymmetric Ge monochromator for a high-intensity $\mathrm{Cu} \mathrm{K} \alpha 1$ beam. A Tecnai F30ST TEM operated at $300 \mathrm{kV}(0.19 \mathrm{~nm}$ point-to-point resolution) was used for the TEM characterization. The cross-section TEM samples were prepared by

TABLE I. A summary of the nominal structural parameters, the growth conditions, and the TDD of the different buffer samples. The substrate temperature was fixed at $475^{\circ} \mathrm{C}$ in all the growth processes.

\begin{tabular}{|c|c|c|c|c|c|c|c|c|c|}
\hline \multirow[b]{2}{*}{ Sample } & \multicolumn{4}{|c|}{ First layer } & \multicolumn{4}{|c|}{ Second layer } & \multirow[b]{2}{*}{$\operatorname{TDD}\left(\mathrm{cm}^{-2}\right)$} \\
\hline & $x$ & $t(\mu \mathrm{m})$ & Type & Growth rate $(\mathrm{nm} / \mathrm{s})$ & $x$ & $t(\mu \mathrm{m})$ & Type & Growth rate $(\mathrm{nm} / \mathrm{s})$ & \\
\hline A & $\ldots$ & $\ldots$ & $\ldots$ & $\ldots$ & 0.75 & 1.0 & Constant composition & 6.0 & $>1.0 \times 10^{9}$ \\
\hline B & 0.4 & 0.5 & Constant composition & 2.0 & 0.75 & 0.5 & Constant composition & 6.0 & $5.0 \times 10^{8}$ \\
\hline $\mathrm{C}$ & 0.4 & 0.5 & Constant composition & 2.0 & $0.4 \rightarrow 0.75$ & 0.5 & Linear grading & $2.0 \rightarrow 1.2$ & $1.7 \times 10^{8}$ \\
\hline $\mathrm{D}$ & $\ldots$ & $\ldots$ & $\ldots$ & $\ldots$ & $0.3 \rightarrow 0.75$ & 1.0 & Linear grading & $10 \rightarrow 6.0$ & $3.3 \times 10^{8}$ \\
\hline
\end{tabular}



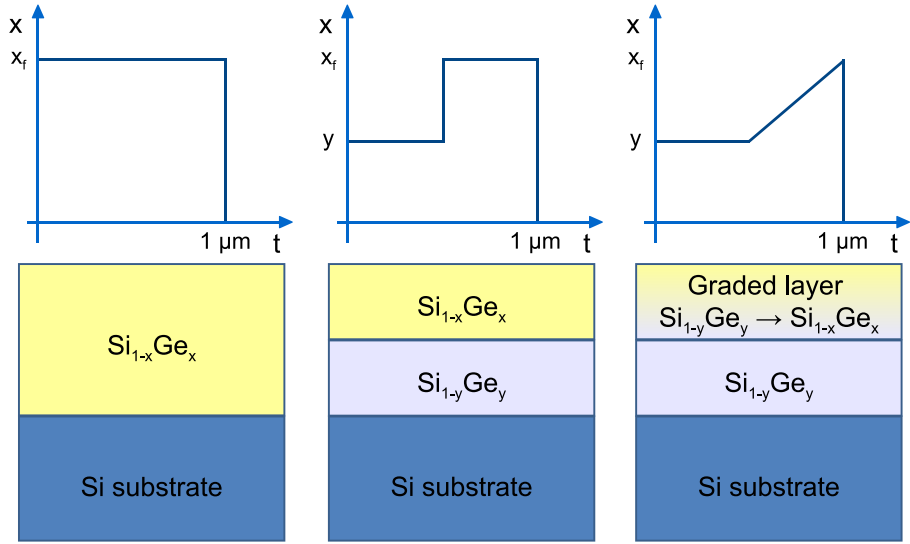

Sample A

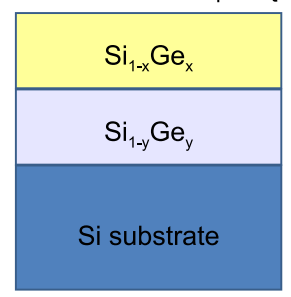

Sample B

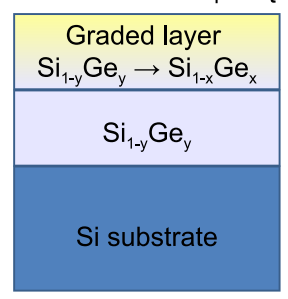

Sample C

mechanical pre-preparation and subsequent Ar-ion milling $\left(4.3 \mathrm{kV}, 4^{\circ}\right)$, the plan view TEM samples were prepared by wet chemical etching. Plan view TEM analysis (averaging over 20 images) was used for the determination of the TDD (see last column in Table I). AFM topography pictures were taken using a Veeco Innova microscope in tapping mode.

The optical analysis of the Ge/SiGe MQWs was performed through room temperature absorption (ABS) and photoluminescence (PL) measurements. ABS spectra were measured in a Jasco V-570 spectrometer with a Peltier cooled $\mathrm{PbS}$ photoresistance; the Brewster geometry was employed in order to strongly reduce thin film interference effects. A Jasco FT/IR-800 Fourier transform spectrometer, equipped with a Peltier cooled $\mathrm{PbS}$ photoresistance (cutoff at $0.4 \mathrm{eV}$ ), was employed for PL measurements. Samples were excited using the $1064 \mathrm{~nm}$ line of a Nd: $\mathrm{YVO}_{4}$ laser, with an exciting power density of about $1 \mathrm{~kW} / \mathrm{cm}^{2}$.

\section{STRUCTURAL CHARACTERIZATION}

Figure 2 summarizes the results of the structural analysis: XRD $\omega-2 \theta$ scans around the (004) reflection, which for symmetrical reflections are along the $Q_{z}$ axis, AFM topography and TEM plan view pictures are reported for each sample.

A surface root-mean-square (rms) roughness below $0.5 \mathrm{~nm}$ was measured for all the samples, guaranteed by the relatively low growth temperature. Cross-hatch roughness is present in all the samples except sample A. The measured Ge content and relaxation of the top layer of the different buffers were found to be comprised between $75.3 \%-76 \%$ and $101 \%-102.5 \%$, respectively. The (004) Bragg peak of the Si substrate is visible at $Q_{z}=4.6276 \AA^{-1}$ for each sample and is used as a reference for the SiGe (004) signals which are visible at lower $Q_{z}$ values. The narrow width of the SiGe peaks in the $Q_{z}$ direction demonstrates the good crystal quality of the grown material.

The XRD $\omega-2 \theta$ scan of sample A, in which the peak at $Q_{z}=4.4918 \AA^{-1}$ corresponds to the SiGe layer, shows the good crystal quality of the layer compared to the Si substrate. No cross-hatch roughness is visible in the AFM picture, underlining the presence of short misfit segments (disordered misfit dislocation network). Indeed, a TDD $>10^{9} \mathrm{~cm}^{-2}$ was measured for this sample.
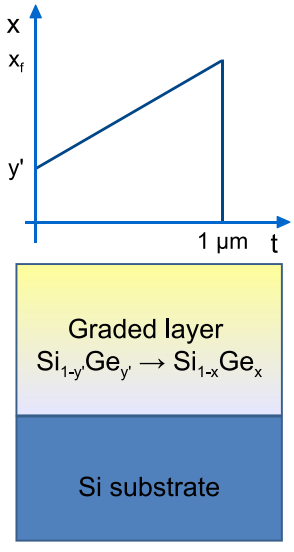

Sample D
FIG. 1. Schematics of the different thin buffers: Sample A (reference buffer), sample B (double step buffer), sample C (double step graded buffer), and sample D (partial graded buffer). Each schematic is accompanied by a Ge content $x$ versus thickness $t$ plot.
The presence of the two SiGe layers with different composition is clearly visible in the XRD $\omega-2 \theta$ scan of sample $\mathrm{B}$, in which the peak at $Q_{z}=4.5523 \AA^{-1}$ corresponds to the lower Ge content layer. The total mismatch to be released is the same as for sample A, but strain partitioning in the two layers prevents an excessive MD nucleation and reduces mutual TD blocking. As a consequence, cross-hatch roughness is present as shown in the AFM picture, while the TDD is reduced to $5 \times 10^{8} \mathrm{~cm}^{-2}$.

The structure of sample $\mathrm{C}$ is as well easily detectable in the corresponding XRD $\omega-2 \theta$ scan: The composition
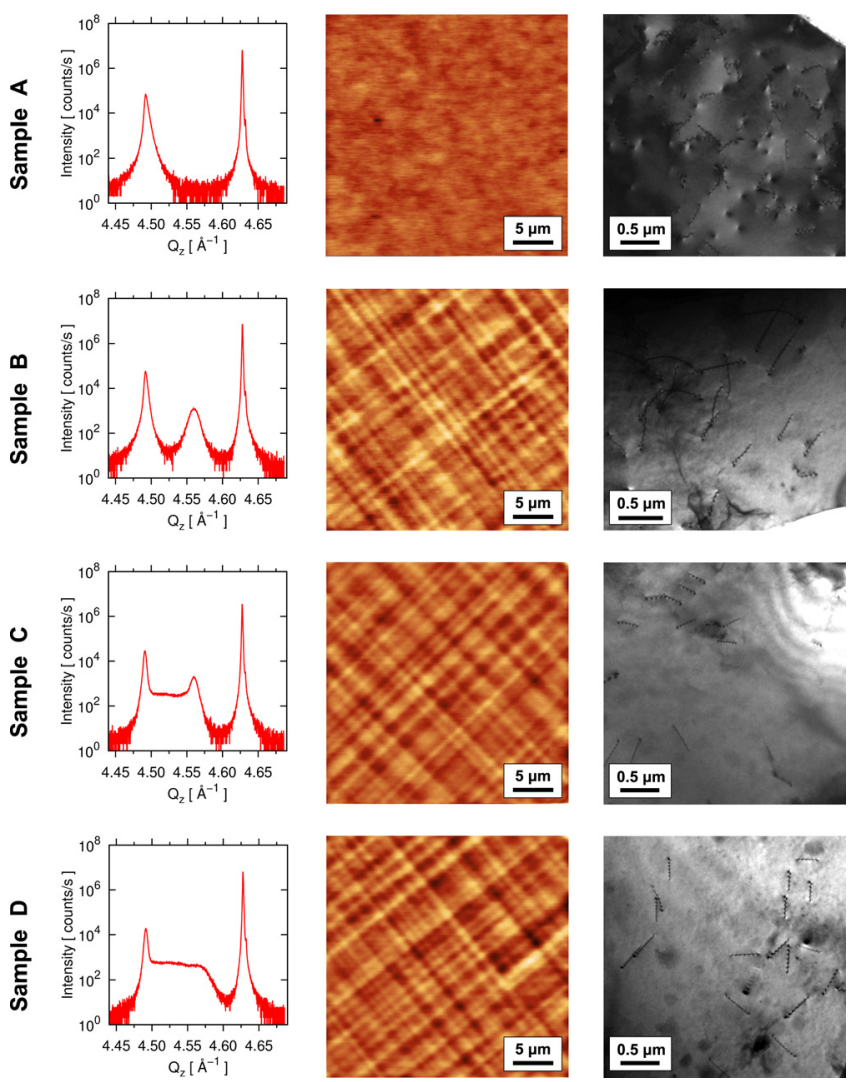

FIG. 2. XRD $\omega-2 \theta$ scans around the (004) reflection, AFM topography, and TEM plan view pictures of the different buffers: Sample A (reference buffer), sample B (double step buffer), sample C (double step graded buffer), and sample D (partial graded buffer). Plan view TEM pictures show the threading dislocations reaching the surface (visible as black spots and dotted line). 
grading gives rise to the roughly constant intensity profile in between the two SiGe peaks. Despite the fast grading rate of $70 \% / \mu \mathrm{m}$, which is $\sim 10$ times larger compared to the one used in standard thick GVS, the introduction of the graded layer still yields a substantial reduction of the TDD to $1.7 \times 10^{8} \mathrm{~cm}^{-2}$.

The XRD $\omega-2 \theta$ scan of sample D presents the intensity profile resulting from the partially graded buffer. The measured TDD $\left(3.3 \times 10^{8} \mathrm{~cm}^{-2}\right)$ is in between those of samples B and $\mathrm{C}$.

All the investigated buffers show a lower TDD when compared to the constant composition reference (sample A). Nevertheless, the most promising approach in terms of TDD reduction is sample $\mathrm{C}$.

A further degree of freedom towards the reduction of TDD can be given by the optimization of the growth parameters. As an example, an additional improvement has been achieved by depositing the graded layer of sample $\mathrm{C}$ at higher growth rates (from $10 \mathrm{~nm} / \mathrm{s}$ to $6 \mathrm{~nm} / \mathrm{s}$ ). The measured TDD for this buffer is $7.8 \times 10^{7} \mathrm{~cm}^{-2}$.

In order to better clarify the mechanism leading to the TDD reduction, in Fig. 3 we compare plan view and cross section TEM images of samples $\mathrm{A}$ and $\mathrm{C}$. The plan view images of the two samples have been already reported in Fig. 2. Each dotted line in plan view images corresponds to a single threading arm and indicates that the dislocation line is not perpendicular to the surface, most likely gliding on (111) planes. Sharp pits in plan view images provide evidence for the presence of a second population of dislocations running perpendicular to the (001) plane. Examples of a dotted line
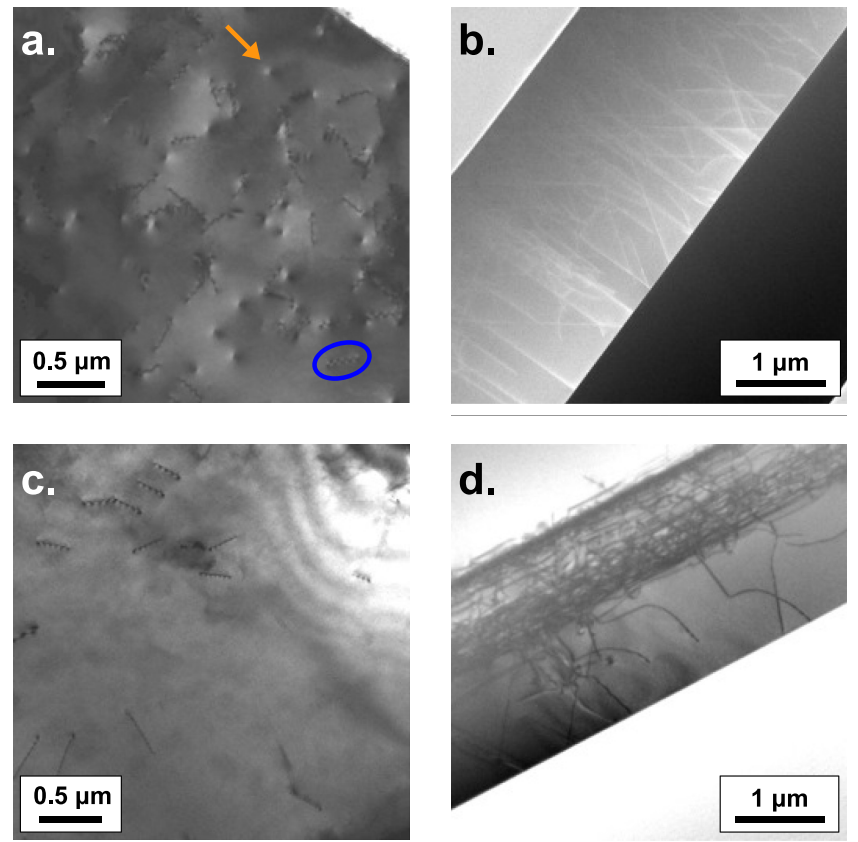

FIG. 3. Plan view and cross section TEM images of sample A ((a) and (b)) and sample $\mathrm{C}((\mathrm{c})$ and (d)). (a), (c), and (d): Bright field TEM images acquired in (220) Bragg condition. (b) Dark field scanning TEM image in (110) zone axis orientation. Dotted lines and sharp pits in plan view images, as the ones underlined in (a) by a blue circle and an orange arrow, correspond to $60^{\circ}$ dislocations and vertical dislocations, respectively. In crossplane images (b) and (d), the top left edge corresponds to the $\mathrm{SiGe} / \mathrm{Si}$ interface. and a sharp pit are underlined in Fig. 3(a) by a blue circle and an orange arrow, respectively. These vertical dislocations are typical of highly mismatched heteroepitaxy (Ref. 27), and their formation is favored by an out-of-equilibrium concentration of high-mobility vacancies, which is easily obtained at high deposition rates and low deposition temperatures. ${ }^{28}$ Since vertical dislocations lie outside the (111) planes, they cannot glide, which greatly reduces the probability of dislocation annihilation and TDD reduction. In sample A, a substantial fraction of threading dislocations runs perpendicular to the interface (sharp pits in Fig. 3(a)). In the case of sample C, only $60^{\circ}$ dislocations are seen in plan view TEM images (see Fig. 3(c)). The formation of such glissile dislocations during the relaxation of the intermediate layer favors the interaction of threading arms during the subsequent deposition of the graded layer leading to a reduced TDD. This analysis is confirmed by the cross section images shown in Figs. 3(b) and 3(d). The dense network of dislocations in (d) corresponds to the $\sim 1 \mu \mathrm{m}$ thick double step graded layer.

In Table II, a representative selection of literature data for high Ge content SiGe buffers is reported, together with the best result presented in this work. Our data can be compared in particular with the works of Shah et al. (Refs. 21 and 23) and Capellini et al. (Ref. 29), both focused on thin relaxed buffers comprising a Ge epilayer interposed between the Si substrate and the $\mathrm{SiGe}$ relaxed buffer. The thin buffers of Refs. 21, 23, and 29 outperform our most representative sample in terms of TDD, yet our double step grading approach provides an extremely low rms roughness and a limited thermal budget, resulting from the low deposition temperature and the short deposition time ( $\sim 10 \mathrm{~min})$.

\section{Ge MQW DEPOSITION AND OPTICAL CHARACTERIZATION}

As a further validation of the effectiveness of the proposed approach, we used the buffer layer characterized by the higher structural quality (sample C) as a VS for the deposition of $\mathrm{Ge} / \mathrm{Si}_{0.15} \mathrm{Ge}_{0.85} \mathrm{MQWs}$ (sample MQWonC). The optical quality of the multilayer has been compared with that of $\mathrm{Ge} / \mathrm{Si}_{0.15} \mathrm{Ge}_{0.85} \mathrm{MQWs}$ that have been grown on a standard $14 \mu \mathrm{m}$ thick GVS (sample MQWonGVS) and on a $2 \mu \mathrm{m}$ thick constant composition $\mathrm{Si}_{0.1} \mathrm{Ge}_{0.9}$ buffer (sample MQWonA). All the MQW samples feature 50 nominally $10 \mathrm{~nm}$ thick Ge quantum wells and $21 \mathrm{~nm}$ thick $\mathrm{Si}_{0.15} \mathrm{Ge}_{0.85}$ barriers. The structure of MQWonGVS sample is analogous to those discussed in Refs. 31-33 and can be used as a benchmark of quality.

The growth of MQW structures requires strain balance between the well and the barrier layers (Ref. 34), which implies a VS with a proper final composition matching the average Ge content of the MQW stack. In the case of the studied $\mathrm{Ge} / \mathrm{Si}_{0.15} \mathrm{Ge}_{0.85} \mathrm{MQWs}$, a VS with a final Ge fraction $x_{f}=90 \%$ is needed. Consequently, a modified version of sample $\mathrm{C}$ has been developed: While the first layer of the buffer was kept unchanged, the second layer was graded from $\mathrm{Si}_{0.6} \mathrm{Ge}_{0.4}$ to $\mathrm{Si}_{0.1} \mathrm{Ge}_{0.9}(100 \% / \mu \mathrm{m})$. This modified buffer reproduced the structural quality of sample $\mathrm{C}$ (as shown by similar structural analysis, not discussed here). An 
TABLE II. Comparison of representative literature data for high Ge content SiGe buffers, together with the best result presented in this work (sample C). The two sets of data from the works of Shah et al. (Refs. 21 and 23) differ in the grading rate of the reverse graded layer.

\begin{tabular}{|c|c|c|c|c|c|c|}
\hline Approach & $\begin{array}{l}\text { Growth } \\
\text { Technique }\end{array}$ & $\begin{array}{c}x_{f} \\
(\%)\end{array}$ & $\begin{array}{l}\text { Total } \\
\text { Thickness } \\
\quad(\mu \mathrm{m})\end{array}$ & $\begin{array}{c}\text { Growth } \\
\text { Temperature } \\
\left({ }^{\circ} \mathrm{C}\right)\end{array}$ & $\begin{array}{c}\mathrm{rms} \\
\text { Roughness } \\
\quad(\mathrm{nm})\end{array}$ & $\begin{array}{l}\text { TDD } \\
\left(\mathrm{cm}^{-2}\right)\end{array}$ \\
\hline Linear grading $^{\mathrm{a}}$ & UHV-CVD & 100 & 12 & 800 & 47 & $(1-5) \times 10^{7}$ \\
\hline Linear grading with $\mathrm{CMP}^{\mathrm{a}}$ & UHV-CVD & 100 & 12 & $800 / 750 / 550$ & 24.2 & $(2.1 \pm 0.2) \times 10^{6}$ \\
\hline Linear grading with $\mathrm{CMP}^{\mathrm{b}}$ & RP-CVD & 75 & 9 & 850 & 15 & $1 \times 10^{5}$ \\
\hline Reverse grading ${ }^{\mathrm{c}}$ & RP-CVD & 78 & 2.8 & $400-670 / 850$ & $2.6 \pm 0.3$ & $(4.2 \pm 0.1) \times 10^{6}$ \\
\hline Reverse grading ${ }^{\mathrm{d}}$ & RP-CVD & 78 & 3.8 & $400-670-830 / 850$ & 1.9 & $3.3 \times 10^{6}$ \\
\hline $\mathrm{SiGe} / \mathrm{Ge} / \mathrm{Si}^{\mathrm{e}}$ & UHV-CVD & 78 & 0.7 & $350-550 / 550$ & 5 & $\sim 10^{7}$ \\
\hline Linear grading $^{\mathrm{f}}$ & LEPECVD & 80 & 12 & $750 \rightarrow 575$ & 2.9 & $3 \times 10^{6}$ \\
\hline Double step grading (sample C) & LEPECVD & 75 & 2 & 475 & $<0.5$ & $7.8 \times 10^{7}$ \\
\hline
\end{tabular}

${ }^{\mathrm{a}}$ Reference 18.

${ }^{\mathrm{b}}$ Reference 19 .

${ }^{\mathrm{c}}$ Reference 21.

${ }^{\mathrm{d}}$ Reference 23.

${ }^{\mathrm{e}}$ Reference 29.

${ }^{\mathrm{f}}$ Reference 30.

${ }^{g}$ Present work.

additional $1 \mu \mathrm{m}$ thick $\mathrm{Si}_{0.1} \mathrm{Ge}_{0.9}$ layer was deposited in order to allow the comparison with MQWs on standard GVSs.

Figure 4 shows the room temperature ABS spectra of samples MQWonC, MQWonGVS, and MQWonA. The stair-case shape typical of the density of states of type-I QWs is clearly visible in all the spectra. The ABS features are due to direct transitions between confined states at $\Gamma$, as discussed in Refs. 31 and 32. Marked excitonic peaks are visible in the spectrum of sample MQWonGVS, they become smoother in the spectrum of sample MQWonC and completely disappear in the spectrum of sample MQWonA. In the following comparison, we will concentrate on the first ABS peak, related to the $\mathrm{HH} 1-\mathrm{c} \Gamma 1$ transition from the first heavy hole to the first conduction band confined states.

The room temperature PL spectra of samples MQWonGVS and MQWonC are reported in Fig. 5. As discussed in Ref. 33, the transition at about $0.85 \mathrm{eV}$ is

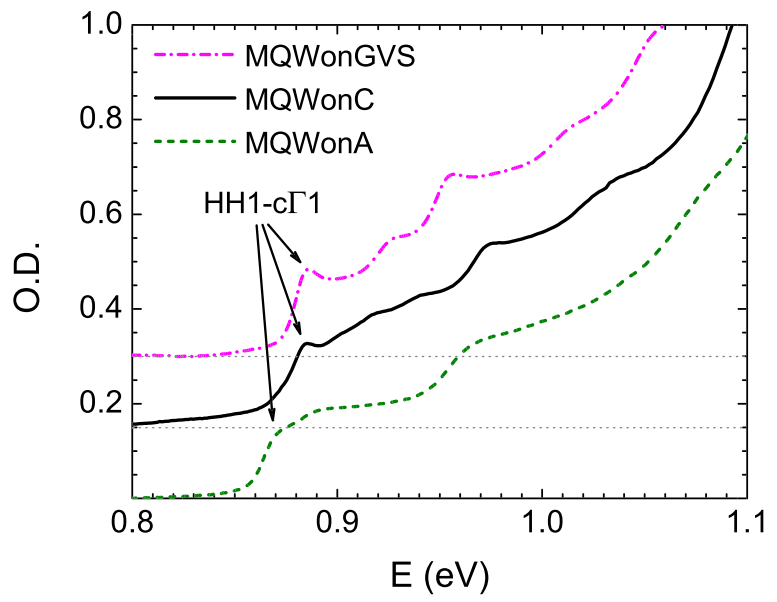

FIG. 4. Room temperature absorption spectra of nominally identical MQWs deposited on double step graded buffer (MQWonC, black continuous line), thick graded buffer (MQWonGVS, purple dashed-dotted line), and constant composition buffer (MQWonA, green dashed line), respectively. The data have been offset for clarity. The slight differences in peak energies are related to the different actual well thicknesses. attributed to the direct $\mathrm{c} \Gamma 1-\mathrm{HH} 1$ recombination, while the broad band at lower energies is due to the indirect cL1-HH1 emission, where cL1 is the first confined state at the L point of the conduction band. The overall PL intensity of sample MQWonC is about 2 times lower compared to that of sample MQWonGVS. The emission of sample MQWonA could not be resolved from the experimental noise and is not reported in the figure.

ABS excitonic lines can be used for an assessment of the structural quality of the QW stack that in turn reflects the quality of the buffer. In fact, threading dislocations are expected to induce the broadening and, eventually, the disappearance of the excitonic peaks. Furthermore, in PL spectra, the emission intensity acts as an indicator of structural quality.

The fact that excitonic peaks are visible at room temperature in the ABS spectrum of MQWonC, together with the observation of interband recombination at room temperature in the PL spectrum, indicates that the structural quality of MQWonC is close to that of MQWonGVS, despite the noticeably reduced thickness of the buffer in the first case.

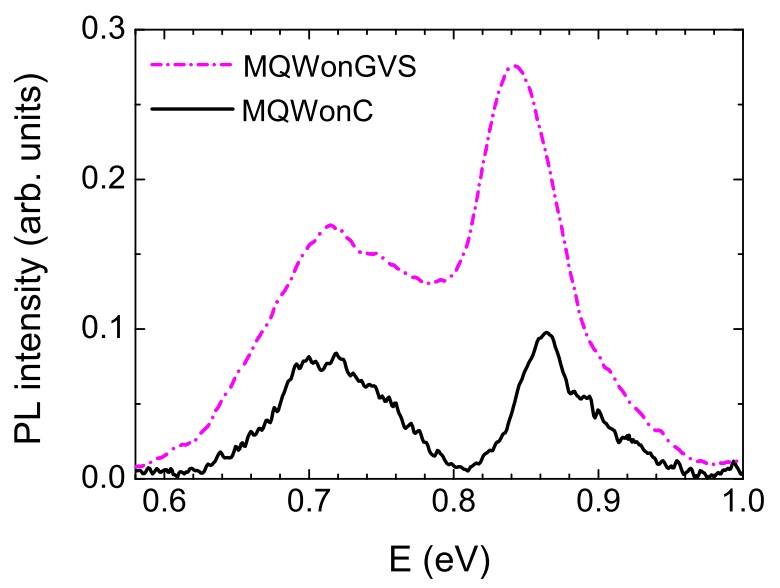

FIG. 5. Room temperature photoluminescence spectra for samples MQWonC (black continuous line) and MQWonGVS (purple dashed-dotted line). 
Nevertheless, half width at half maximum values for the $\mathrm{HH} 1-\mathrm{c} \Gamma 1$ transition have been extracted from ABS spectra, obtaining $8 \mathrm{meV}$ and $11 \mathrm{meV}$ for samples MQWonGVS and MQWonC, respectively.

On the other hand, the use of a constant composition VS (sample MQWonA) results detrimental in terms of structural and optical quality, as suggested by the disappearance of excitonic peaks in the ABS spectrum and by the very low emitted intensity which prevents the detection of the PL spectrum. These evidences confirm the results of the structural analysis presented in Sec. III.

\section{CONCLUSIONS}

We studied different thin Ge-rich SiGe buffers grown by LEPECVD meant to be virtual substrates for the integration of $\mathrm{Ge}$ heterostructures on $\mathrm{Si}$. The chosen growth temperature guarantees a low thermal budget, making our approach appealing for CMOS integration. All the proposed designs demonstrate a reduction of the TDD compared to constant composition SiGe films having the same thickness and final Ge content. In particular, the double step grading approach allows a reduction of the TDD up to $7.8 \times 10^{7} \mathrm{~cm}^{-2}$, which can be explained in terms of dislocation dynamics and control of the growth parameters. The effectiveness of this design was confirmed by the optical analysis of $\mathrm{Ge} / \mathrm{SiGe}$ MQWs grown on top of a double step graded buffer: Their optical quality is close to that typical of MQWs grown on standard thick GVSs, despite the buffer total thickness has been reduced by a factor of 7 . Indeed, exciton ABS peaks are visible also at room temperature, and the overall PL intensity is only 2 times lower.

\section{ACKNOWLEDGMENTS}

The authors would like to thank the EC ICT FET Proactive Initiative Towards Zeropower ICT for funding the research through the GREEN Silicon Project (No. 257750).

${ }^{1}$ C. O. Chui, H. Kim, D. Chi, B. B. Triplett, P. C. McIntyre, and K. C. Saraswat, Tech. Dig. - Int. Electron Devices Meet. 2002, 437.

${ }^{2}$ Y.-H. Kuo, Y. K. Lee, Y. Ge, S. Ren, J. E. Roth, T. I. Kamins, D. A. B. Miller, and J. S. Harris, Nature 437, 1334 (2005).

${ }^{3}$ Y. Busby, M. De Seta, G. Capellini, F. Evangelisti, M. Ortolani, M. Virgilio, G. Grosso, G. Pizzi, P. Calvani, S. Lupi, M. Nardone, G. Nicotra, and C. Spinella, Phys. Rev. B 82, 205317 (2010).

${ }^{4}$ L. Lever, Y. Hu, M. Myronov, X. Liu, N. Owens, F. Y. Gardes, I. P. Marko, S. J. Sweeney, Z. Ikonić, D. R. Leadley, G. T. Reed, and R. W. Kelsall, Opt. Lett. 36, 4158 (2011).

${ }^{5}$ P. Chaisakul, D. Marris-Morini, G. Isella, D. Chrastina, M.-S. Rouifed, J. Frigerio, and L. Vivien, Solid State Electron. 83, 92 (2013).

${ }^{6}$ F. Pezzoli, F. Bottegoni, D. Trivedi, F. Ciccacci, A. Giorgioni, P. Li, S. Cecchi, E. Grilli, Y. Song, M. Guzzi, H. Dery, and G. Isella, Phys. Rev. Lett. 108, 156603 (2012).
${ }^{7}$ S. Cecchi, T. Etzelstorfer, E. Müller, A. Samarelli, L. F. Llin, D. Chrastina, G. Isella, J. Stangl, and D. J. Paul, J. Mater. Sci. 48, 2829 (2013).

${ }^{8}$ A. Samarelli, L. F. Llin, S. Cecchi, J. Frigerio, T. Etzelstorfer, E. Müller, Y. Zhang, J. R. Watling, D. Chrastina, G. Isella, J. Stangl, J. P. Hague, J. M. R. Weaver, P. Dobson, and D. J. Paul, J. Appl. Phys. 113, 233704 (2013).

${ }^{9}$ J. G. Fiorenza, G. Braithwaite, C. W. Leitz, M. T. Currie, J. Yap, F. Singaporewala, V. K. Yang, T. A. Langdo, J. Carlin, M. Somerville, A. Lochtefeld, H. Badawi, and M. T. Bulsara, Semicond. Sci. Technol. 19, L4 (2004).

${ }^{10}$ L. M. Giovane, H.-C. Luan, A. M. Agarwal, and L. C. Kimerling, Appl. Phys. Lett. 78, 541 (2001)

${ }^{11}$ J. R. Watling and D. J. Paul, J. Appl. Phys. 110, 114508 (2011).

${ }^{12}$ J. W. Matthews, J. Vac. Sci. Technol. 12, 126 (1975).

${ }^{13}$ Y. B. Bolkhovityanov, O. P. Pchelyakov, M. A. Revenko, L. V. Sokolov, and S. I. Chikichev, Semiconductors 37, 493 (2003).

${ }^{14}$ E. A. Fitzgerald, Y.-H. Xie, M. L. Green, D. Brasen, A. R. Kortan, J. Michel, Y.-J. Mii, and B. E. Weir, Appl. Phys. Lett. 59, 811 (1991).

${ }^{15}$ M. Kummer, C. Rosenblad, A. Dommann, T. Hackbarth, G. Höck, M. Zeuner, E. Müller, and H. von Känel, Mater. Sci. Eng., B 89, 288 (2002).

${ }^{16}$ E. A. Fitzgerald, Y.-H. Xie, D. Monroe, P. J. Silverman, J. M. Kuo, A. R. Kortan, F. A. Thiel, and B. E. Weir, J. Vac. Sci. Technol. B 10, 1807 (1992).

${ }^{17}$ C. Rosenblad, J. Stangl, E. Müller, G. Bauer, and H. von Känel, Mater. Sci. Eng., B 71, 20 (2000).

${ }^{18}$ M. T. Currie, S. B. Samavedam, T. A. Langdo, C. W. Leitz, and E. A. Fitzgerald, Appl. Phys. Lett. 72, 1718 (1998).

${ }^{19}$ Y. Bogumilowicz, J. M. Hartmann, C. D. Nardo, P. Holliger, A.-M. Papon, G. Rolland, and T. Billon, J. Cryst. Growth 290, 523 (2006).

${ }^{20}$ S. Marchionna, A. Virtuani, M. Acciarri, G. Isella, and H. von Kaenel, Mater. Sci. Semicond. Process. 9, 802 (2006).

${ }^{21}$ V. A. Shah, A. Dobbie, M. Myronov, D. J. F. Fulgoni, L. J. Nash, and D. R. Leadley, Appl. Phys. Lett. 93, 192103 (2008).

${ }^{22}$ A. Dobbie, M. Myronov, R. J. H. Morris, A. H. A. Hassan, M. J. Prest, V. A. Shah, E. H. C. Parker, T. E. Whall, and D. R. Leadley, Appl. Phys. Lett. 101, 172108 (2012).

${ }^{23}$ V. A. Shah, A. Dobbie, M. Myronov, and D. R. Leadley, J. Appl. Phys. 107, 064304 (2010).

${ }^{24}$ E. Feltin, B. Beaumont, M. Laügt, P. de Mierry, P. Vennéguès, H. Lahrèche, M. Leroux, and P. Gibart, Appl. Phys. Lett. 79, 3230 (2001).

${ }^{25}$ M. Sakai, T. Egawa, M. Hao, and H. Ishikawa, Jpn. J. Appl. Phys., Part 1 43, 8019 (2004)

${ }^{26}$ D. Chrastina, G. Isella, M. Bollani, B. Rössner, E. Müller, T. Hackbarth, E. Wintersberger, Z. Zhong, J. Stangl, and H. von Känel, J. Cryst. Growth 281, 281 (2005).

${ }^{27}$ A. Marzegalli, F. Isa, H. Groiss, E. Müller, C. V. Falub, A. G. Taboada, P. Niedermann, G. Isella, F. Schäffler, F. Montalenti, H. von Känel, and L. Miglio, Adv. Mater. 25, 4408 (2013).

${ }^{28}$ F. Isa, A. Marzegalli, A. G. Taboada, C. V. Falub, G. Isella, F. Montalenti, H. von Känel, and L. Miglio, APL Mater. 1, 052109 (2013).

${ }^{29}$ G. Capellini, M. De Seta, Y. Busby, M. Pea, F. Evangelisti, G. Nicotra, C. Spinella, M. Nardone, and C. Ferrari, J. Appl. Phys. 107, 063504 (2010).

${ }^{30}$ D. Chrastina, B. Rössner, G. Isella, H. von Känel, J. P. Hague, T. Hackbarth, H.-J. Herzog, K.-H. Hieber, and U. König, in Materials for Information Technology, edited by E. Zschech, C. Whelan, and T. Mikolajick (Springer, 2005), pp. 17-29.

${ }^{31}$ M. Bonfanti, E. Grilli, M. Guzzi, M. Virgilio, G. Grosso, D. Chrastina, G. Isella, H. von Känel, and A. Neels, Phys. Rev. B 78, 041407(R) (2008).

${ }^{32}$ M. Bonfanti, E. Grilli, M. Guzzi, D. Chrastina, G. Isella, H. von Känel, and H. Sigg, Physica E 41, 972 (2009).

${ }^{33}$ E. Gatti, E. Grilli, M. Guzzi, D. Chrastina, G. Isella, and H. von Känel, Appl. Phys. Lett. 98, 031106 (2011).

${ }^{34}$ D. J. Paul, Laser Photonics Rev. 4, 610 (2010). 